RESEARCH ARTICLE - BEES

\title{
Social Information in the Stingless Bee, Trigona corvina Cockerell (Hymenoptera: Apidae): The Use of Visual and Olfactory Cues at the Food Site
}

\author{
FMJ SOMMERLANDT ${ }^{1}$, W HUBER $^{2}$, J SPAETHE ${ }^{1}$ \\ 1 - University of Würzburg, Würzburg, Germany \\ 2 - University Vienna, Vienna, Austria
}

\section{Article History}

\section{Edited by}

Tom Wenseleers, Katholieke Universiteit Leuven - Belgium

Received 14 September 2014

Initial acceptance 02 November 2014

Final acceptance 01 December 2014

\section{Keywords}

social information, communication, odor marks, visual cues, recruitment.

\section{Corresponding author}

Frank M. J. Sommerlandt

Department of Behavioral Physiology

and Sociobiology, Biozentrum

University of Würzburg, Am Hubland, 97074 Würzburg, Germany

E-Mail: frank.sommerlandt@uni-wuerzburg.de

\begin{abstract}
For social insects, colony performance is largely dependent on the quantity and quality of food intake and thus on the efficiency of its foragers. In addition to innate preferences and previous experience, foragers can use social information to decide when and where to forage. In some stingless bee (Meliponini) species, individual foraging decisions are shown to be influenced by the presence of social information at resource sites. In dual choice tests, we studied whether visual and/or olfactory cues affect individual decision-making in Trigona corvina Cockerell and if this information is species-specific. We found that $T$. corvina foragers possess local enhancement: they are attracted by olfactory and visual cues released by conspecifics but avoid feeders associated with heterospecific individuals of the species Tetragona ziegleri (Friese). Overall, olfactory cues seem to be more important than visual cues, but information by visual cues alone is sufficient for discrimination.
\end{abstract}

\section{Introduction}

When foragers of eusocial insects approach new food sources, their decision to land can be influenced by the presence or absence of visual or chemical information provided by other individuals (Slaa et al., 2003; Danchin et al., 2004; Leadbeater \& Chittka, 2007; Yokoi \& Fujisaki, 2011). For example, foragers can either be attracted (local enhancement) or repelled (local inhibition) by the presence of conspecifics (von Frisch, 1914; D'Adamo et al., 2000; Slaa et al., 2003).

Social information in general is any information about behavior, physical presence or remnant provided by a sender that reduces uncertainty of a receiver. The information can be produced on purpose or inadvertently and may be of different modalities, as for example visual, olfactory or tactile (Danchin et al., 2004; Dall et al., 2005; Kendal et al., 2005; Gruter \& Leadbeater, 2014). Such publicly accessible information provided by conspecific or heterospecific individuals allows the observer to adaptively change its behavior to gain fitness benefits, such as assessing the location and quality of a food source (Chittka \& Leadbeater, 2005; Goodale et al., 2010, Yokoi \& Fujisaki, 2011). In bees, olfactory information at the food source can be provided by foragers through anal droplets, gland secretions or footprints originating from glandular epithelia of the claw retractor tendon (Nieh et al., 2003; Jarau et al., 2004; Barth et al., 2008; Wilms \& Eltz, 2008; Jarau, 2009). In bumblebees and honeybees, for example, scent marks can be either short-lived and repellent, and used to avoid visitation of a recently depleted nectar source, or longer-lasting and attracting, and allows identification of particularly rewarding flowers (Stout \& Goulson, 2001). The response to such marks depends also on the bee's previous experience (Hrncir et al., 2009; Saleh \& Chittka, 2006) and can be modified through learning (reviewed in Gruter \& Leadbeater, 2014). Moreover, chemical information is also provided by the profile of epicuticular hydrocarbons, which is species- and colony-specific (Howard \& Blomquist, 1982; Nunes et al., 2011).

Another potential modality depicts the physical presence of conspecifics or heterospecifics that serves as a visual stimulus. 
In social wasps, for example, visual information about resident heterospecific wasps at the feeder site can appear attractive or repellent to foraging individuals, depending on characteristics of the involved species (size, aggressiveness, etc.; Raveret Richter $\&$ Tisch, 1999). The use of conspecific and heterospecific visual information for decision-making in foragers is also reported for bumblebees (Stout \& Goulson, 2001; Dawson \& Chittka, 2012), which can learn to use this information to find profitable food sources (Dawson \& Chittka, 2012). Under natural conditions, signals and cues from different modalities are used to gain social information about the food source. Findings in the stingless bee Scaptotrigona mexicana (Guérin) indicate a redundant function of visual and chemical cues in the bee's recruitment system at the food site (Sánchez et al., 2011). In general, in stingless bees the response to the presence or absence of nestmates or heterospecifics at the food site is usually species specific (Slaa et al., 2003).

The stingless bee Trigona corvina Cockerell plays an important role in plant pollination in the neotropics, since pollen from more than 70 different plant species has been found in a single colony (Roubik \& Moreno Patiño, 2009). Known to be an aggressive mass-recruiter (Roubik, 1981), the fitness of a $T$. corvina colony relies on an efficient recruitment system, where nestmates are guided to profitable food sources mainly by field-based mechanisms including pheromone trails and deposition of scent marks near the food source (Aguilar et al., 2005; Jarau et al., 2010). T. corvina foragers are known to be attracted to the food source by scent marks deposited by themselves (during previous visits), by nestmates or by conspecific non-nestmates, but no evidence was found for the use of heterospecific odor marks (Boogert et al., 2006). Whether $T$. corvina foragers also rely on visual cues provided by the presence of conspecifics and if there is a cross-effect (e.g. additive, redundant or hierarchical) of visual and chemical cues that affect the forager's decision at the food site, needs to be investigated. Here we aim to evaluate the significance of chemical and visual cues on the choice behavior of T. corvina foragers. We tested the significance of the presence of conspecific or heterospecific foragers, the effect of epicuticular hydrocarbons and the impact of conspecific forager-deposited odor marks.

In particular, we addressed the following questions:

i) Do foragers of $T$. corvina possess local enhancement or local inhibition when making decisions at food sites?

ii) Which sensory modality (olfaction or vision) is used for decision-making?

iii) Can foragers use visual cues to discriminate between conspecifics and heterospecifics?

\section{Material and Methods}

Experiments were performed in March 2013 (experiments 1, 5, 6) and March 2014 (experiments 1, 2, 3, 4, 5) at the Tropical Research Station La Gamba, Golfito, Costa Rica (www.univie.ac.at/ lagamba/). Workers of the stingless bee T. corvina were trained to a gravity feeder(Spaethe et al., 2014) providing 0.1 M sucrose solution. A small sub-group of bees (up to 10-15 individuals) was then trained from the central feeder to a second feeder that was placed approx. 8-10 $\mathrm{m}$ away and that provided $0.3 \mathrm{M}$ sucrose solution. This training procedure allowed us to work with a constant number of foragers during the experiments. The second feeder was made of a piece of foam material $(85 \mathrm{~mm} \times 85 \mathrm{~mm} \times 10 \mathrm{~mm})$ with a central hole $(15$ $\mathrm{mm}$ in diameter) covered with filter paper (Fig. 1A). A snap-on cap with sucrose solution (or water during the test) was placed into the hole. This setup allowed a (olfactory) marking of the filter paper by visiting bees (see Fig. 1B; comparable to Sánchez et al., 2011). The experimental procedure consisted of two phases: a training phase and a test phase. During the training phase, the above mentioned second feeder (hereinafter referred to as training feeder) provided a sugar reward, and the bees were allowed to forage for 10 minutes to establish the food source. The first training phase of each day was prolonged to 60 minutes to obtain a stable number of foraging bees. Afterwards, during the test phase, a choice test was performed where the bees had to choose between two new, unrewarded feeders (comparable to Boogert et al., 2006) that provided different olfactory and/or visual cues according to the type of experiment (see results for the tested combinations). These feeders, consequently termed test feeders, were placed at $30 \mathrm{~cm}$ distance between each other. Each bee was individually tested and immediately captured after landing on one of the test feeders to avoid multiple counting. After the decisions of six bees, the test feeders were removed and replaced by a fresh rewarding training feeder to start a new experiment. Following a 10 minute training phase, a new randomly chosen combination of test feeders was presented and the next six bees were tested. During all experiments, the feeder positions were randomized to avoid position effects. We used different types of feeders: To test visual cues, we attached three hexane-washed conspecific individuals (Fig. 1A) or individuals from the related species Tetragona ziegleri (Friese). T. ziegleri was chosen since it is of similar size as $T$. corvina (about $6 \mathrm{~mm}$ body length), but of different color (orange). Thus, both species should be detectable by $T$. corvina foragers at a similar distance, but provide different chromatic information. The hexane-bath was applied for 24 hours and hexane was regularly exchanged to remove all species-specific odors. Before the dummies were used in an experiment, they were sundried for 60 minutes. To assess the impact of epicuticular hydrocarbons, we attached three freshly killed (by freezing) bees. We used olfactory marked feeders with filter papers that were odormarked by foragers in previous visits to test for the relevance of odor markings of the feeder. The filter papers were marked by 10-15 foragers which completed multiple foraging bouts during the 10 minute training phases (see below). Although we never observed active pheromone deposition by the bees through abdominal droplets, we cannot completely exclude pheromone marking. We chose randomly among a couple of marked filter papers for the ones that were used in the tests. Overall, not more than 30 minutes passed between deposition of odor marks and the use in the experiments. For the control experiment, we used a new and empty control feeder covered with clean filter paper. 
Experiments were performed in a randomized order to avoid temporal effects and with inter-test intervals of 10 minutes to obtain stable numbers of recruited foragers at the feeders. This time period corresponded to approximately four foraging flights per individual. We assume all tested bees belonged to a single colony, since no inter-individual aggression behavior was observed at the feeders and T. corvina foragers are known to defend food sources against competitors (Roubik, 1981). All captured bees were released at the end of a day. As a consequence, we cannot exclude that some bees were counted multiple times during the following days.

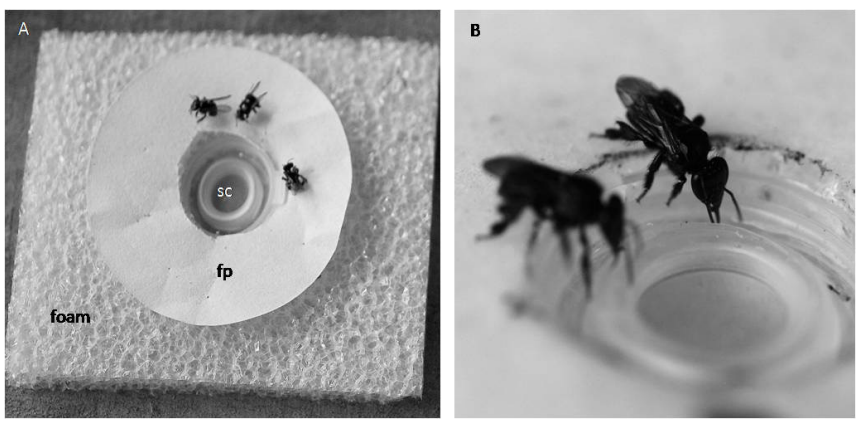

Fig 1. Training and test feeder. A, Feeder with pinned bee dummies. $\mathrm{B}$, Bees feeding on a training feeder which allowed for the deposition of odor marks on the filter paper. fp, filter paper; sc, snap-on cap.

The proportion of choices for all observed landings was calculated for each of the feeder types and shown as bar charts. To calculate the statistics, we used the observed number of landings on either feeder and compared it with the null hypothesis, which is a random choice (1:1 distribution on the two test feeders), using chi-square test. Statistics were performed with IBM ${ }^{\circledR}$ SPSS $®$ Statistics software (Version 20).

\section{Results}

Altogether, we counted 450 decisions in 6 different feeder combinations (Fig. 2).

To test if foragers of T. corvina possess local enhancement or local inhibition at the feeder site, bees had to choose between a feeder with three freshly killed conspecifics and a clean feeder (experiment 1$)$. The majority of bees $(83 \%)$ preferred the feeder with the (freshly killed) conspecifics compared to the plain feeder $\left(p<0.001, \mathrm{chi}^{2}=24.000\right)$. To evaluate if odor cues, e.g. epicuticular hydrocarbons play a role in the recognition of nestmates at the feeding site, or if the visual presence of conspecifics is sufficient for the effect of local enhancement, we confronted the foraging bees with one feeder surrounded by three freshly killed conspecifics and a second feeder surrounded by the same number of hexane-washed dead conspecifics (experiment 2). Significantly more foragers landed on the feeder occupied by freshly killed conspecifics $(68 \%)$ compared to the feeder with odorless dummies $\left(32 \% ; \mathrm{p}=0.034, \mathrm{chi}^{2}=4.513\right)$. In experiment 3 , we tested if T. corvina foragers can distinguish between conspecifics and heterospecifics (both freshly killed). In this test, conspecifics were more attractive $(70 \%)$ than heterospecifics of the species $T$. ziegleri $\left(\mathrm{p}=0.021, \mathrm{chi}^{2}=5.328\right)$. We then eliminated the epicuticular hydrocarbons by washing the dummies of both species with hexane, with the result that the tested foragers could only use visual cues to discriminate between conspecifics and heterospecifics (experiment 4). Both species were similar in body size (approx. $6 \mathrm{~mm}$ body length) but differed in their chromatic appearance with $T$. corvina being almost entirely black and T. ziegleri possessing a mainly orange-colored body (Jarau et al., 2009). Foragers significantly preferred conspecifics over heterospecifics. $68 \%$ of the tested bees decided to land on the feeder occupied by three hexane-washed T. corvina, compared to $32 \%$ of the foragers that landed in the vicinity of $T$. ziegleri $(\mathrm{p}=0.034$, $\operatorname{chi}^{2}=4.513$ ).

We then tested the impact of conspecific odor marks (on filter paper) on recruited foragers (experiment 5). Odor marks alone were significantly more attractive $(72 \%)$ than clean feeders $\left(p=0.002\right.$, chi $\left.^{2}=9.649\right)$. In experiment 6 , we presented two attractive cues in a competition experiment: one feeder hosting hexane-washed conspecifics, whereas the second feeder provided a filter paper containing odor marks released by conspecifics. Foragers possessed a preference towards the odor marks $(76 \%)$, compared to the visual presence of conspecifics $\left(\mathrm{p}=0.032, \mathrm{chi}^{2}=4.593\right)$.

To test for side-specific preferences of the bees, we also presented two clean feeders and found that bees showed no side-preference but chose randomly between both feeders ( $\mathrm{p}=0.300, \mathrm{chi}^{2}=1.086, \mathrm{~N}=30$; data not shown).

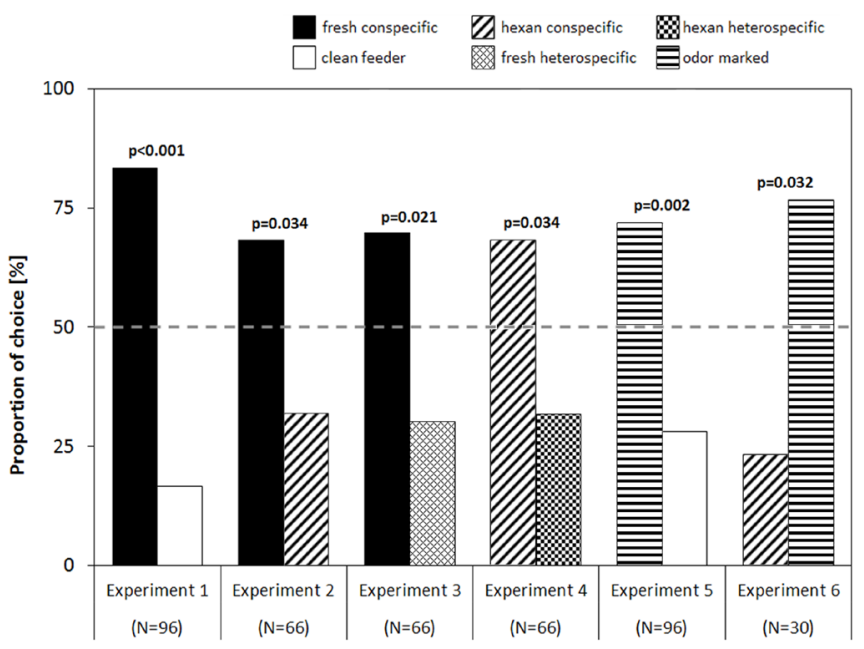

Fig 2. Mean proportion of choices made by Trigona corvina foragers towards each of the two feeders offered in each experiment. For each test combination, p-values are shown above the bars. For statistics see text. Numbers in brackets indicate number of choices, dashed line indicates random choice level.

\section{Discussion}

Foragers of T. corvina use social information when deciding to land on an established food source and make use of olfactory and visual information. In accordance with findings by Boogert et al. (2006), T. corvina foragers possess local enhancement, i.e. newly arriving foragers prefer 
to land close to other conspecifics (a possible distinction between local enhancement and stimulus enhancement and its relevance is reviewed in Heyes, 1994 and was recently discussed in Avarguès-Weber \& Chittka, 2014). Our species is described as relatively aggressive (Johnson \& Hubbel, 1974; Roubik, 1981); foragers often arrive at resources in groups and defend their foraging territories (Roubik, 1981). Following nestmates to a profitable food source could ensure the monopolization of the food source. T. corvina can make use of visual and chemical cues when orienting towards a food source that bears species-specific information, but odor marks seem to be more attractive than the visual presence of a nestmate, as already reported for another stingless bee species, S. mexicana (Sánchez et al., 2011).

As previously shown for olfactory cues (Boogert et al., 2006), T. corvina foragers can discriminate conspecifics from heterospecifics by their visual appearance alone. In our experiments, feeders surrounded by freshly killed conspecifics - providing the visual information of the presence of the bees combined with the species specific profile of epicuticular hydrocarbons - were preferred by the recruited bees over clean feeders and hexane-washed conspecifics or heterospecifics (experiments 1-3). Thus, the information of cuticular hydrocarbon profiles to recognize nestmates, as shown for several other stingless bee species (Buchwald \& Breed, 2005; Nunes et al., 2008; Ferreira-Caliman et al., 2010), plays a role in the recruitment behavior at the food site, as well. Interestingly, conspecifics were also recognized and preferred over heterospecifics when the cuticular hydrocarbon profile and other species-specific odors were absent (due to hexane-washing), indicating that visual information alone is sufficient to identify and discriminate conspecifics from heterospecifics (experiment 4).

Both species used in our experiment were of approximately the same size (about $6 \mathrm{~mm}$ body length; Jarau et al., 2009) but differed in color, with $T$. corvina being almost entirely black and T. ziegleri possessing a mainly orange-colored body. Thus, aside from flower recognition (Spaethe et al., 2014), color vision may also play an important role in species discrimination at resource sites in stingless bees. Our results concur with findings in other species of the genus Trigona (Villa \& Weiss, 1990; Spaethe et al., 2014), whereby the relevance of visual cues for decision-making at the food site was reported and the impact of visual cues for individual recognition could have been demonstrated for social wasps (Raveret Richter \& Tisch, 1999; Sheehan \& Tibbetts, 2011). Whether $T$. corvina foragers can identify heterospecifics that differ from conspecifics in size but not in color, needs to be investigated. When confronted with competing information (experiment 6 ), olfactory marks (on the filter paper) were preferred over visual cues of hexane-washed bees. We assume the olfactory marks in our experiment to be footprints secreted from the leg tips, as footprints were reported as a type of scent marks at the food source for other stingless bees (Melipona seminigra Friese: Hrncir et al., 2004, Melipona scutellaris Latreille: Hrncir et al., 2009). Additionally, we did not observe any deposition of abdominal droplets.

The efficacy of scent marks at the food source (experiment 5) was also shown in T. corvina (Boogert et al., 2006) and other stingless bee species (Sánchez et al., 2011) and emphasizes the importance of olfaction as the major sensory modality. This could be caused by either a bias in sensitivity for visual and olfactory stimuli, a difference in the stimuli's action range or a true (innate) preference of the bees for scent marks over visual stimuli. Except for the non-volatile longchained epicuticular hydrocarbons, odor marks can act over larger distances than visual cues. Estimates of spatial visual resolution in the stingless bee Tetragonisca angustula (Latreille) which with 5-6 mm body length is about the size as T. corvina, suggest that objects of the size of conspecifics should be visually detectable at relatively short distances of approx. 10 to $20 \mathrm{~cm}$ (Zeil \& Wittmann, 1993). In contrast, forager-deposited odor marks can be sensed by conspecific stingless bees over long distances up to 10 to $20 \mathrm{~m}$ (Melipona panamica Cockerell: Nieh, 1998; Scaptotrigona aff. depilis (Moure): Schmidt et al., 2003).

Here we report that foragers of the stingless bee $T$. corvina possess local enhancement at the food site and that they use visual cues in addition to, or in the absence of, olfactory marks, to identify conspecifics. Whether these bees can also visually discriminate more similar heterospecifics, such as the similar-sized and colored Trigona fuscipennis Friese, needs to be investigated.

\section{Acknowledgements}

We thank F. Bötzl, J. Kriegbaum, D. Materna and A. Weiglein for help with data collection, S. Jarau for species identification, and the Tropical Research Station La Gamba (www.univie.ac.at/lagamba/), Costa Rica, for making available their laboratory facilities and tropical garden. The Costa Rican Ministerio de Ambiente y Energía kindly granted research permits. We further thank two annonymous reviewers for their thoughtful comments on an earlier draft of this manuscript and J. Plant for linguistic advice. This work was supported by a $\mathrm{PhD}$ research scholarship offered by the Free State of Bavaria (Elitenetzwerk Bayern) to FMJS.

\section{References}

Aguilar, I., Fonseca, A. \& Biesmeijer, J. C. (2005). Recruitment and communication of food source location in three species of stingless bees (Hymenoptera, Apidae, Meliponini). Apidologie, 36: 313-324. DOI: 10.1051/apido:2005005

Avarguès-Weber, A. \& Chittka, L. (2014). Local enhancement or stimulus enhancement? Bumblebee social learning results in a specific pattern of flower preference. Anim. Behav., 185-191. DOI: 10.1016/j.anbehav.2014.09.020 
Barth, F. G., Hrncir, M. \& Jarau, S. (2008). Signals and cues in the recruitment behavior of stingless bees (Meliponini). J Comp Physiol A ,194: 313-327. DOI: 10.1007/s00359-008-0321-7

Boogert, N. J., Hofstede, F. E. \& Aguilar Monge, I. (2006). The use of food source scent marks by the stingless bee Trigona corvina (Hymenoptera: Apidae): the importance of the depositor's identity. Apidologie, 37: 366-375. DOI: 10.1051/ apido:2006001

Buchwald, R. \& Breed, M. D. (2005). Nestmate recognition cues in a stingless bee, Trigona fulviventris. Anim. Behav., 70: 1331-1337. DOI: 10.1016/j.anbehav.2005.03.017

Chittka, L. \& Leadbeater, E. (2005). Social learning: public information in insects. Curr. Biol., 15(21): R869-871. DOI: 10.1016/j.cub.2005.10.018

D’Adamo, P., Corley, J., Sackmann, P. \& Lozada, M. (2000). Local enhancement in the wasp Vespula germanica - Are visual cues all that matter? Insectes soc., 47: 289-291

Dall, S. R., Giraldeau, L. A., Olsson, O., McNamara, J. M. \& Stephens, D. W. (2005). Information and its use by animals in evolutionary ecology. Trends Ecol. Evol., 20: 187-193. DOI: 10.1016/j.tree.2005.01.010

Danchin, E., Giraldeau, L.-A., Valone, T. J. \& Wagner, R. H. (2004). Public information: From nosy neighbors to cultural evolution. Science, 305: 487-491

Dawson, E. H. \& Chittka, L. (2012). Conspecific and heterospecific information use in bumblebees. PLoS ONE 7(2): e31444 DOI: 10.1371/journal.pone.0031444

Ferreira-Caliman, M. J., Nascimento, F. S., Turatti, I. C., Mateus, S., Lopes, N. P. \& Zucchi, R. (2010). The cuticular hydrocarbons profiles in the stingless bee Melipona marginata reflect task-related differences. J Insect. Physiol., 56: 800-804 DOI: 10.1016/j.jinsphys.2010.02.004

Goodale, E., Beauchamp, G., Magrath, R. D., Nieh, J. C. \& Ruxton, G. D. (2010). Interspecific information transfer influences animal community structure. Trends Ecol. Evol., 25: 354-361 DOI: 10.1016/j.tree.2010.01.002

Gruter, C. \& Leadbeater, E. (2014). Insights from insects about adaptive social information use. Trends Ecol. Evol., 29: 177-184 DOI: 10.1016/j.tree.2014.01.004

Heyes, C. M. (1994). Social learning in animals: categories and mechanisms. Biol. Rev., 69: 207-231

Howard, R. W. \& Blomquist, G. J. (1982). Chemical ecology and biochemistry of insect hydrocabons. Annu. Rev. Entomol., 27: 149-172

Hrncir, M., Jarau, S., Zucchi, R. \& Barth, F. G. (2004). On the origin and properties of scent marks deposited at the food source by a stingless bee, Melipona seminigra. Apidologie, 35: 3-13 DOI: 10.1051/apido:2003069
Hrncir, M., Roselino, A. C., Rodrigues, A. V. \& Zucchi, R. (2009). Stingless bee footprints at the food sources - repellents, attractants or both? Proceedings of the 46th Annual Meeting of the Animal Behavior Society. Pirenópolis, Brazil: 90-91.

Jarau, S. (2009). Chemical communication during food exploitation in stingless bees. In S. Jarau \& M. Hrncir (Eds.). Food Exploitation by Social Insects: Ecological, Behavioral, and Theoretical Approaches (pp. 223-249). Boca Raton (FL), CRC Press.

Jarau, S., Dambacher, J., Twele, R., Aguilar, I., Francke, W. \& Ayasse, M. (2010). The trail pheromone of a stingless bee, Trigona corvina (Hymenoptera, Apidae, Meliponini), varies between populations. Chem. Senses, 35: 593-601. DOI: 10.1093/ chemse/bjq057

Jarau, S., Hrncir, M., Ayasse, M., Schulz, C., Francke, W., Zucchi, R. \& Barth, F. G. (2004). A stingless bee (Melipona seminigra) marks food sources with a pheromone from its claw retractor tendons. J. Chem. Ecol., 30: 793-804

Jarau, S., Morawetz, L., Reichle, C., Gruber, M. H., Huber, W. \& Weissenhofer, A. (2009). Corbiculate Bees of the Golfo Dulce Region, Costa Rica. Wien, Verein zur Förderung der Tropenstation La Gamba, Costa Rica.

Johnson, L. K. \& Hubbel, S. P. (1974). Aggression and competition among stingless bees: field studies. Ecology, 55: 120-127

Kendal, R. L., Coolen, I., van Bergen, Y. \& Laland, K. N. (2005). Trade-offs in the adaptive use of social and asocial learning. Adv. Study Behav., 35: 333-379. DOI: 10.1016/ s0065-3454(05)35008-x

Leadbeater, E. \& Chittka, L. (2007). The dynamics of social learning in an insect model, the bumblebee (Bombus terrestris). Behav. Ecol. Sociobiol., 61: 1789-1796. DOI: 10.1007/ s00265-007-0412-4

Nieh, J. C. (1998). The role of a scent beacon in the communication of food location by the stingless bee, Melipona panamica. Behav. Ecol. Sociobiol., 43: 47-58

Nieh, J. C., Ramirez, S. \& Nogueira-Neto, P. (2003). Multisource odor-marking of food by a stingless bee, Melipona mandacaia. Behav. Ecol. Sociobiol., 54: 578-586 DOI: 10.1007/s00265-003-0658-4

Nunes, T. M., Mateus, S., Turatti, I. C., Morgan, E. D. \& Zucchi, R. (2011). Nestmate recognition in the stingless bee Frieseomelitta varia (Hymenoptera, Apidae, Meliponini): sources of chemical signals. Anim. Behav. 81: 463-467. DOI: 10.1016/j.anbehav.2010.11.020

Nunes, T. M., Nascimento, F. S., Turatti, I. C., Lopes, N. P. \& Zucchi, R. (2008). Nestmate recognition in a stingless bee: does the similarity of chemical cues determine guard acceptance? Anim. Behav., 75: 1165-1171. DOI: 10.1016/j.anbehav.2007.08.028

Raveret Richter, M. \& Tisch, V. L. (1999). Resource choice 
of social wasps: influence of presence, size and species of resident wasps. Insectes Soc., 46: 131-136

Roubik, D. W. (1981). Comparative foraging behavior of Apis mellifera and Trigona corvina (Hymenoptera: Apidae) on Baltimora recta (Compositae). Rev. Biol. Trop., 29: 177183

Roubik, D. W. \& Moreno Patiño, J. E. (2009). Trigona corvi$n a$ : An ecological study based on unusual nest structure and pollen analysis. Psyche: A Journal of Entomology, 2009: Article ID 268756, 7 pages. DOI: 10.1155/2009/268756

Saleh, N. \& Chittka, L. (2006). The importance of experience in the interpretation of conspecific chemical signals. Behav. Ecol. Sociobiol., 61: 215-220. DOI: 10.1007/s00265-006-0252-7

Sánchez, D., Nieh, J. C. \& Vandame, R. (2011). Visual and chemical cues provide redundant information in the multimodal recruitment system of the stingless bee Scaptotrigona mexicana (Apidae, Meliponini). Insectes Soc., 58: 575-579 DOI: $10.1007 / \mathrm{s} 00040-011-0181-y$

Schmidt, V. M., Zucchi, R. \& Barth, F. G. (2003). A stingless bee marks the feeding site in addition to the scent path (Scaptotrigona aff. depilis). Apidologie, 34: 237-248. DOI: 10.1051/apido:2003021

Sheehan, M. J. \& Tibbetts, E. A. (2011). Specialized face learning is associated with individual recognition in paper wasps. Science, 334(6060): 1272-1275. DOI: $10.1126 /$ science. 1211334
Slaa, E. J., Wassenberg, J. \& Biesmeijer, C. (2003). The use of field-based social information in eusocial foragers: local enhancement among nestmates and heterospecifics in stingless bees. Ecol. Entomol., 28: 369-379

Spaethe, J., Streinzer, M., Eckert, J., May, S. \& Dyer, A. G. (2014). Behavioural evidence of colour vision in free flying stingless bees. J. Comp. Physiol. A, 200: 485-496. DOI: 10.1007/s00359-014-0886-2

Stout, J. C. \& Goulson, D. (2001). The use of conspecific and interspecific scent marks by foraging bumblebees and honeybees. Anim. Behav., 62: 183-189 DOI: 10.1006/anbe.2001.1729

Villa, J. D. \& Weiss, M. R. (1990). Observations on the use of visual and olfactory cues by Trigona spp foragers. Apidologie, 21: $541-545$

von Frisch, K. (1914). Der Farbensinn und der Formensinn der Biene. Jena, Verlag von Gustav Fischer.

Wilms, J. \& Eltz, T. (2008). Foraging scent marks of bumblebees: footprint cues rather than pheromone signals. Naturwissenschaften, 95: 149-153. DOI: 10.1007/s00114-007-0298-Z

Yokoi, T. \& Fujisaki, K. (2011). To forage or not: Responses of bees to the presence of other bees on flowers. Ann. Entomol. Soc. Am., 104: 353-357. DOI: 10.1603/an10053

Zeil, J. \& Wittmann, D. (1993). Landmark orientation during the approach to the nest in the stingless bee Trigona (Tetragonisca) angustula (Apidae, Meliponinae). Insectes Soc., 40: 381-389 